\title{
Demand Side Participation for Frequency Containment in the Web of Cells Architecture
}

\author{
M. H. Syed and G. M. Burt \\ Institute for Energy and Environment \\ University of Strathclyde, \\ Glasgow, Scotland \\ mazheruddin.syed@strath.ac.uk
}

\author{
J. K. Kok \\ Smart Electricity Grids \\ TNO \\ The Hague, The Netherlands \\ koen.kok@tno.nl
}

\author{
R. D'Hulst \\ VITO, EnergyVille \\ Boeretang 200 \\ 2400 Mol, Belgium \\ reinhilde.dhulst@vito.be
}

\begin{abstract}
A large number of demand side management schemes have been proposed in literature for provision of frequency control ancillary services to the network. However, it is assumed that all the flexible devices within the network are managed and controlled under one demand side management (DSM) scheme. In this paper, two independent demand side management schemes control the portfolio of flexible devices within a web of cells architecture. A methodology and scenarios for analysis of the performance of more than one DSM scheme within the same network have been realized using a real-time power hardware-in-the-loop co-simulation platform, and the paper presents this as a basis for investigations of such arrangements.
\end{abstract}

Keywords-Ancillary services, demand side management, transactive energy markets and real-time power hardware-in-theloop simulation.

\section{INTRODUCTION}

$\mathrm{A}^{\mathrm{N}}$ NCILLARY services in the electrical power system are essential to maintaining the integrity and stability of transmission and distribution systems [1]. Ancillary services can be broadly classified into three: (i) Frequency Control Ancillary Services (FCAS), (ii) Network Control Ancillary Services (NCAS) and (iii) System Restart Ancillary Services (SRAS) [2]. Ensuring sufficient procurement and prompt delivery of ancillary services is one of the main responsibilities of a system operator, and, hence, account for a large portion of their budget. Great Britain's Transmission System Operator (TSO), National Grid, for instance paid a total amount of over $£ 30$ million (47 million US dollar) to ensure the availability and provision of ancillary services during the month of August 2014 alone [3]. The ongoing transition to a sustainable energy system has led to an increase of intermittent renewable generation and decommissioning of conventional fossil fuel based generation. Increased supplyside intermittency increases the need for ancillary services, while at the same time the traditional ancillary service capacity diminishes. Hence, alternative methods to ancillary service provision are being sought by TSOs.

Traditionally, ancillary services are delivered by large generators, while the demand side is used only in critical situations when fast acting generators are no longer able to stabilize the grid. In these cases, blunt measures such as interrupting blocks of load by means of under-frequency relays are a common practice. With the advancements in information and control technology, initiatives have been taken to involve industrial/commercial customers to participate in ancillary service delivery [4]. However, the full potential of demand response is sorely untapped.

Demand response (DR) refers to the shifting of demand by consumers, actively by behavioural changes or passively by means of automation, in response to a provided incentive. Any program that encompasses the principle of DR is referred to as a demand side management (DSM) scheme. The shift in demand is obtained most of the time by means of flexible devices. The best candidates for flexible devices are the loads whose utility to the consumer is a function of energy consumed over a period of time rather than instantaneous power consumption (for example: heating, cooling and pumping loads) [5]. The cyclic nature of these loads enables short interruptions that would be acceptable to the consumer. As for the control philosophy of demand, it can be categorized as either centralized or decentralized. In centralized control, the power consumption of the loads can be manipulated automatically or manually (either by utilities, aggregators or customers) in response to a signal received from a center responsible for its operation and control [6]-[9]. In contrast, decentralized control refers to a control in which the power consumption of the loads is manipulated automatically or manually by local measurements and settings [10]-[15]. Further, a mix of centralized and decentralized control is also possible where the power consumption of the devices corresponds to both the central signal and the local measurement and settings [16], [17].

There are primarily three pools of reserves in which demand side can participate to provide frequency support to the network: 1) Frequency Containment Reserve, 2) Frequency Restoration reserve and 3) Frequency Replacement Reserves. Frequency containment reserve refers to the pool of reserve that can be utilized instantaneously in order to contain the frequency after an event based on local measurements or information, whereas frequency restoration refers to the support that will begin at a fixed time (say every quarter of an hour) upon instruction from the system operator with the aim to restore the frequency back to its nominal value. In this 
paper, the focus will be on the former. A large number of advanced DSM schemes for provision of dynamic frequency support have been presented in literature, however these assumed that there is only one DSM scheme present in the entire network. The future power network as envisioned would provide an open platform for utilization of multiple schemes. An important research question remains unanswered in relation to the ability of multiple DSM schemes to co-exist in the same network under similar or different control objectives.

This paper presents a plan for the performance analysis of two independent instances of DSM schemes operating within an experimental network under similar objectives, and an assessment of associated implications. In order to do so, a DSM scheme has been implemented in ELECTRA's (European Liaison on Electricity Committed Towards longterm Research Activity) Web of Cells architecture for the first time [18]. Two independent aggregators with DR participate in the network with the objective of providing dynamic frequency support to the network. Two cases for provision of dynamic frequency support to the network have been investigated. A real-time power hardware-in-the-loop co-simulation platform is hosted at the University of Strathclyde's Distribution Network and Protection (D-NAP) laboratory to experimentally evaluate the feasibility of dynamic frequency provision.

\section{Web of Cells ArChitecture AND Demand Side MANAGEMENT SCHEME}

The web of cells architecture, as shown in Fig. 1., was introduced by ELECTRA. A cell is defined as a group of loads and generation resources, interconnected at same or multiple voltage levels, within a well-defined electrical and geographical boundary. Each cell is connected to its neighbors by means of one or more tie lines. The tie lines may or may not provide the ability of global system re-configuration. Each cell can have an operator responsible for its secure operation. A group of cells is managed and operated by a Control Cell Operator. The aim of the web of cells architecture is to utilize the inter-cell coordination capability to support a secure system-wide operation. The ELECTRA web of cells architecture provides a cornerstone for the investigation of the performance of multiple DSM schemes within a network.

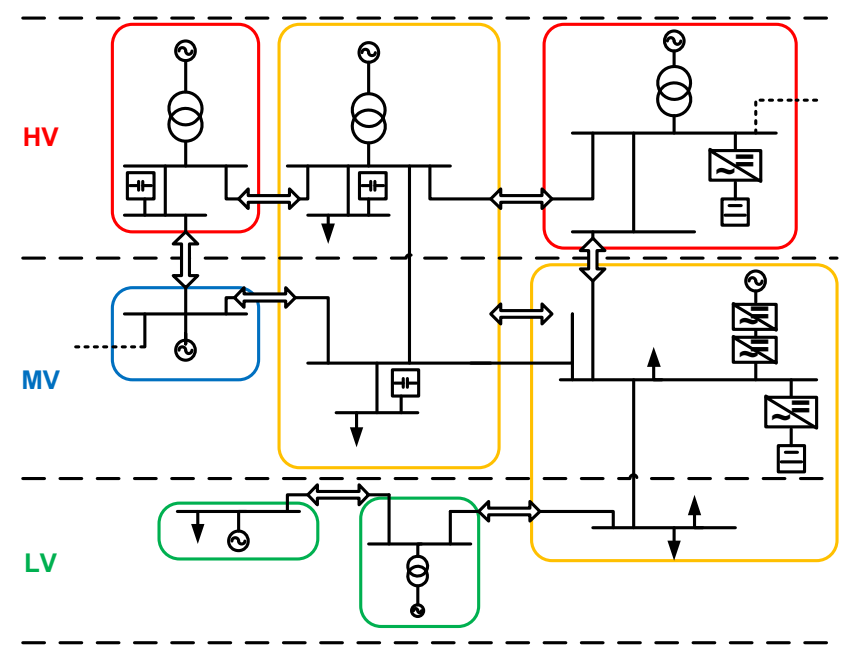

Fig. 1. ELECTRA web of cells architecture
PowerMatcher has been utilized as the DSM scheme for this work. PowerMatcher coordinates a cluster of electricity producing and consuming devices by creating a transactive energy market [19]. It exploits the capability of multi-agent system framework to implement scalable, distributed, complex and open Information and Communication Technology infrastructures. In a multi-agent based environment, theoretically, multiple software agents interact and negotiate to reach a common system goal while preserving their local goal. Similarly, in a PowerMatcher cluster, the interest of each device, either producing or consuming electricity, is represented by a device agent. A centralized auctioneering agent facilitates the device agents to trade in the transactive energy market. Each device in the cluster sends a bid to the auctioneering agent. The bid is indicative of the priority or willingness of the device to consume or produce electricity at a given price. The auctioneering agent aggregates the bid and a market clearing price is determined from the aggregated bid. The market clearing price is then communicated back to the device agents, which depending upon their bid either start consuming or producing electricity or wait till the market price/device priority changes. The only information that is communicated from the device agents to the auctioneering agent is the bid and price from auctioneering agent to the device agents.

\section{METHOdOLOGY AND SCENARIOS FOR EVALUATION}

In order to investigate the performance of multiple DR technologies, a test system with three cells has been chosen as shown in Fig. 2 for the experiment. Two cells out of the three are controlled and operated by two independent demand side aggregators. The two aggregators use independent instances of PowerMatcher technology as the DSM scheme to maintain power balance within its own cell and to participate in frequency containment reserve. The two individual instances of the PowerMatcher technology are considered as the two individual DSM schemes (although the technology is same, the instances are independent of each other with different portfolios). The two cells managed and operated by the demand side aggregators are referred to as the controlled cells. The third cell is defined as an uncontrolled cell, and has no DR technology. The proportion of DR penetration is chosen arbitrarily as $33 \%$, representing a minority of flexible load.

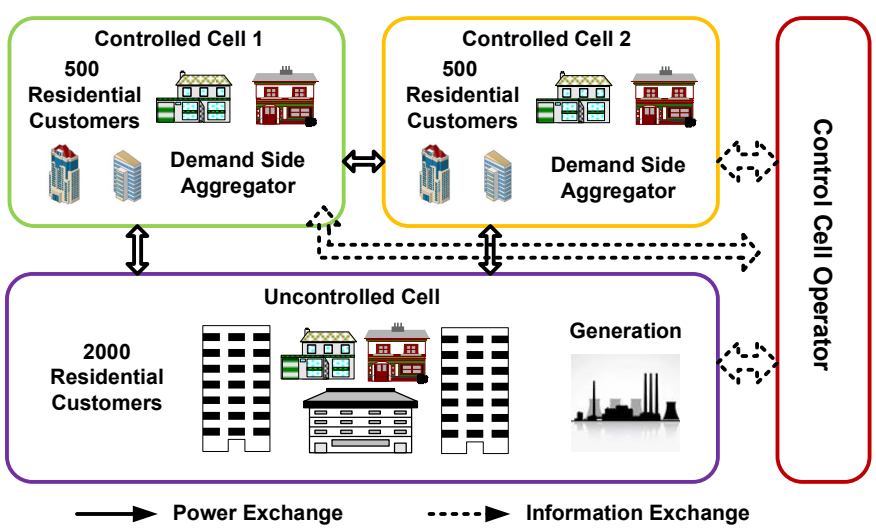

Fig 2. System of cells under study. 
The uncontrolled cell accommodates 2000 residential customers and each controlled cell serves 500 residential houses. Although any cell could host generation, for simplicity in this preliminary work generation is only within the uncontrolled cell. This work is focused on a small test system to allow scrutiny of the interactions, however it will inform later larger implementations. Two alternative measurement modes have been developed for the evaluation of DSM participation in frequency containment and are explained in the subsections below.

\section{A. Local Measurement based Operation}

In this mode of operation, the provision of frequency support is based on local measurements, i.e., measurements available within the cell. Each demand side aggregator of the two controlled cells has access to the frequency measurements within its cell. The operation of flexible devices in the controlled cell is based on the price signal received from the auctioneer and not based on local frequency measurements. Therefore, the frequency measurement aids the decision making process of the PowerMatcher (influences the price). A local measurement based operation algorithm has been developed for this purpose. It is assumed that the frequency of the cell is made available to the cell's PowerMatcher and the algorithm is executed every time step of PowerMatcher. No other system wide information is made available to the individual cell. The overall aim of the algorithm is to manipulate (either turn on or off) the least possible load in order to maintain the frequency within operational limits.

\section{B. Phasor Measurement Unit based Operation}

In this mode of operation, phasor measurement units (PMUs) are present in the network (both controlled and uncontrolled cell) and the measurements are accessible by control cell operator (this assumes the web of cells architecture maintains a centralized control center that would be responsible for monitoring the secure operation of the network and provide additional defensive controls). Valuable measurements of the network are available at the control center and can be used for processing/estimating valuable information. The total active power imbalance in the network is estimated centrally and can be passed on to individual cell controllers based upon their capability to provide the support or on prior agreed volume of support.

\section{EXPERIMENTAL SETUP}

The experimental setup involves two parts, 1) the formation of PowerMatcher cluster simulations and 2) the configuration of a real-time power hardware-in-the-loop co-simulation platform.

\section{A. PowerMatcher Cluster Simulations}

On a day-ahead basis, the demand side aggregator operating in each cell needs to provide an estimated load profile to the network operator. This is utilized by the system network operator for determining the generator scheduling for the coming day. The parameters of each PowerMatcher cluster have been presented in Table I.

A day-ahead estimated load profile has been obtained for this work [20]. The flexible load profile (based on heat pumps here) has been generated in the PowerMatcher simulation tool. To generate the day-ahead estimate for the flexible loads, these loads were operated using a standard on/off temperature control. Each heat pump will heat the water from $30{ }^{\circ} \mathrm{C}$ to $45{ }^{\circ} \mathrm{C}$ (for controlled cell $1,30{ }^{\circ} \mathrm{C}$ to $40{ }^{\circ} \mathrm{C}$ for

Table I

PowerMatcher Load Cluster Parameters for each PM Cell

\begin{tabular}{|c|c|c|c|}
\hline \multirow{2}{*}{$\begin{array}{l}\text { Number } \\
\text { of Houses }\end{array}$} & \multicolumn{2}{|c|}{ Number of } & Capacity of Heat \\
\hline & Bedrooms & Occupants & $\begin{array}{l}\text { Pump Installed } \\
\qquad(\mathrm{kW})\end{array}$ \\
\hline
\end{tabular}

\begin{tabular}{|c|c|c|c|}
\hline 60 & 1 & $\ddot{*}$ & 1.5 \\
\hline 140 & 2 & 率 & 2 \\
\hline 210 & 3 & $\boldsymbol{M}$ & 3 \\
\hline 70 & 4 & $\overrightarrow{M+*}$ & 5 \\
\hline 20 & 5 & $\mathbf{M * *}$ & 5 \\
\hline \multicolumn{2}{|c|}{ Total } & 500 & \\
\hline \multicolumn{4}{|c|}{ Flexible Load Parameters } \\
\hline \multicolumn{2}{|c|}{ Cluster } & Flexible Load & $\begin{array}{c}\text { Temperature } \\
\text { Buffer }\left({ }^{\circ} \mathrm{C}\right) \\
\end{array}$ \\
\hline \multicolumn{2}{|c|}{ Controlled cell 1} & Space Heating & $30-45$ \\
\hline \multicolumn{2}{|c|}{ Controlled cell 2} & Space Heating & $30-40$ \\
\hline
\end{tabular}

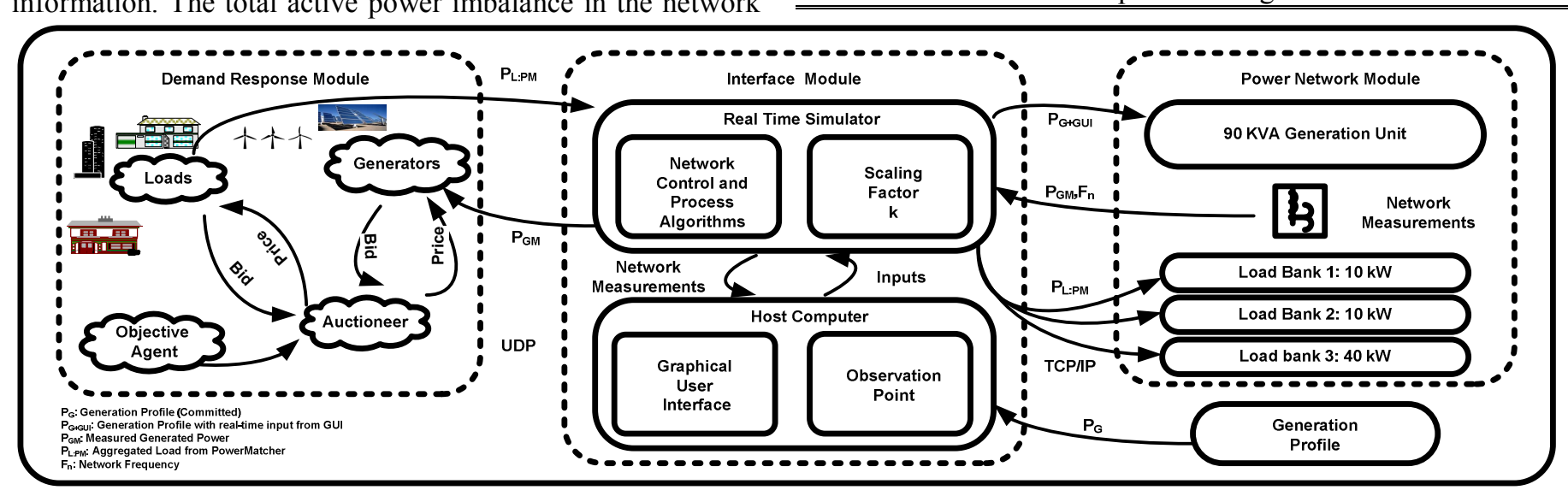

Fig 3. Real-time power hardware-in-the-loop co-simulation platform architecture. 
controlled cell 2) and then wait until the water temperature falls back to $30{ }^{\circ} \mathrm{C}$ before turning on again. This method is sufficient to give diverse behavior but could readily be adapted for other control schemes.

\section{B. Real-Time Power Hardware-in-the-loop Co-simulation Platform}

The DNAP laboratory micro-grid comprises of $80 \mathrm{kVA}$ and 2kVA synchronous generators, 4 induction machines (2X7.5kW, $5.5 \mathrm{~kW}$ and $2.2 \mathrm{~kW}$ ) capable of operating as motor or generators, $15 \mathrm{kVA}$ back to back Triphase inverter unit, 10 kVA 3-phase inverter unit and $60 \mathrm{~kW}$ load banks $(2 \mathrm{X} 10 \mathrm{~kW}$ and $1 \mathrm{X} 40 \mathrm{~kW})$ [21]. To this has been added demand side management capability in the form of two new modules, giving an architecture (presented in Fig. 3) comprising of 1) Power Network module, 2) Demand Response module and 3) an interface module. The architecture of the D-NAP laboratory micro-grid allows operation of the system on the whole as a grid-connected system, and allows formation of three selfsustaining islands. The DR module serves as a plug and play facility, where any DSM scheme can be incorporated for evaluation and testing. In this work, a Matlab implementation of PowerMatcher (PM Simulator) has been incorporated in the DR module. PM simulator runs on a PowerMatcher host computer, and allows modelling of more than one instance of PowerMatcher within the same host computer.

As the name suggests, the third module acts as an interface between the power network module, the DR module and the outside world (including users and other modules). To enable real-time hardware-in-the-loop simulations, the interface module accommodates two real-time simulator units (RTS) from Applied Dynamics International (ADI). Two host computers run the Advantage Framework that provides a feature-rich environment that facilitates designing, building, executing, interacting, visualizing and analyzing the real-time projects. All the algorithms for control and observation of the micro-grid module are based in the interface unit. The RTS processes all the measurements obtained from the micro-grid module and makes them accessible. The communication between the power network module and the interface module is based on TCP/IP. The communication between the interface module and the DR module is based on User Datagram Protocol (UDP). Communication with the outer world is limited to UDP in RTS.

\section{PRELIMINARY EXPERIANCE}

The aim of this work is to perform early experimental investigations of the performance of multiple DSM schemes in frequency support provision to a power system utilizing a web of cells architecture. For each of the two measurement modes described in this paper, two sequencing modes have been developed. In the first sequencing mode, both the controlled cells are synchronized with respect to their response time. The time step chosen for PM simulation is one minute, i.e., a response can be achieved from PowerMatcher every one minute. All the flexible loads that are connected to PowerMatcher will synchronize their control action with the PM simulator "clock". For example, if PM simulator is set up to respond every minute at 12:00, 12:01, 12:02, etc., any device that will be switched on in between 12:00 - 12:01 will be on till 12:01 and then will synchronize its response with the PM simulation time (i.e. 12:02, 12:03, etc.). In the second sequencing mode, the two instances of PowerMatcher are not in synchronism. Although the PM simulator time step is one minute, a 30 second time difference in execution of the two instances of PowerMatcher is maintained. To make it clear, if the PM simulation of controlled cell 1 responds at 12:00:00, 12:01:00, 12:02:00, etc. the PM simulation of controlled cell 2 responds at 12:00:30, 12:01:30, 12:02:30, etc. The two sequencing modes have been chosen to investigate their impact on the network and to assess the associated implications with each mode.

In order to create a frequency contingency in the network, a $500 \mathrm{~kW}$ generation loss is emulated in the test network. This is done in real time with the help of the GUI as mentioned earlier (a scaling factor is present in the interface unit). The modified generation represents the actual generation that is available in the network. The initial experiments show that both the measurement modes are capable of restoring the frequency of the network in real-time. The real-time co-simulation platform has enabled the investigation of power system challenges faced with the emerging DSM schemes. Detailed analysis of the results will be presented in the follow up paper. Future work involves the evaluation of more complex DSM schemes and their interactions within the network, to address any associated implications and extract best practices were possible.

\section{CONCLUSIONS}

In this paper, two instances of a DSM scheme managing a portfolio of flexible loads and participating in the provision of frequency containment within the same network has been presented. The methodology and the scenarios for the evaluation has been presented. The ELECTRA web of cells architecture has been chosen to demonstrate the capability of inter-cell support. Real-time power hardware-in-the-loop platform provides the first hand insight into the implications that might arise from such a control mechanism. The analysis of the performance of the two instances of DSM schemes with the objective of providing frequency support to the network will be presented in the follow up paper.

\section{ACKNOWLEDGMENT}

The work in this paper has been in part supported by the European Commission, under the ELECTRA REX researcher exchange programme of the FP7 project ELECTRA (grant no: 609687). Any opinions, findings and conclusions or recommendations expressed in this material are those of the authors and do not necessarily reflect those of the European Commission. Support has also been provided by TNO under ERP Program "Energy Storage and Conversion".

\section{REFERENCES}

[1] "Monthly Balancing Services Summary, August 2014", [Online] Available: $\quad$ http://www2.nationalgrid.com/UK/Industryinformation/Electricity-transmission-op.erational-data/Reportexplorer/Services-Reports/

[2] "Guide to ancillary services in the national electricity market", [Online] Available: http://www.aemo.com.au/Electricity/MarketOperations/Ancillary-Services. 
[3] F. C. Schweppe , R. D. Tabors , J. L. Kirtley , H. R. Outhred , F. H. Pickel and A. J. Cox "Homeostatic utility control", IEEE Trans. Power App. Syst., vol. PAS-99, pp.1151 -1163, 1980.

[4] "Create new revenue", [Online] Available: http://www.flexitricity.com/connect-your-business

[5] J. A. Short, D. G. Infield, and L. L. Freris, "Stabilization of grid frequency through dynamic demand control," IEEE Trans. Power Syst., vol. 22, no. 3, pp. 1284-1293, Aug. 2007.

[6] Z. Xu, J. Ostergaard, andM. Togeby, "Demand as frequency controlled reserve," IEEE Trans. Power Syst., vol. 26, no. 3, pp. 1062-1071, Aug. 2011.

[7] Douglass, P.J., Garcia-Valle, R.; Nyeng, P.; Ostergaard, J.; Togeby, M., "Smart Demand for Frequency Regulation: Experimental Results", IEEE Trans. Smart Grid, vol.4, no.3, pp.1713,1720, Sept. 2013

[8] A. Molina-Garcia , F. Bouffard and D. S. Kirschen "Decentralized demand-side contributionto primary frequency control", IEEE Trans. PowerSyst., vol. 26, no. 1, pp.411 -4192011

[9] Pourmousavi Kani, S.A.; Nehrir, M.H., "Real-time central demand response for primary frequency regulation in microgrids," Innovative Smart Grid Technologies (ISGT), 2013 IEEE PES , vol., no., pp.1,1, 2427 Feb. 2013

[10] Kondoh, J.; Ning Lu; Hammerstrom, D.J., "An Evaluation of the Water Heater Load Potential for Providing Regulation Service," Power Systems, IEEE Transactions on , vol.26, no.3, pp.1309,1316, Aug. 2011.

[11] F. J. Peterson , J. E. Patton , M. E. Miller , R. A. Gillman , W. M. Warwick and W. F. Sandusky "End-use load and consumer assessment program", Energy Build., vol. 19, no. 3, pp.159 -166 1993

[12] Ning Lu; Chassin, D.P., "A state-queueing model of thermostatically controlled appliances," Power Systems, IEEE Transactions on , vol.19, no.3, pp.1666,1673, Aug. 2004
[13] Hao, H.; Sanandaji, B.M.; Poolla, K.; Vincent, T.L., "Aggregate Flexibility of Thermostatically Controlled Loads," Power Systems, IEEE Transactions on , vol.30, no.1, pp.189,198, Jan. 2015

[14] He Hao; Yashen Lin; Kowli, A.S.; Barooah, P.; Meyn, S., "Ancillary Service to the Grid Through Control of Fans in Commercial Building HVAC Systems," Smart Grid, IEEE Transactions on , vol.5, no.4, pp.2066,2074, July 2014

[15] Lin, Y., Barooah, P., Meyn, S. and Middelkoop, T., "Experimental Evaluation of Frequency Regulation From Commercial Building HVAC Systems", IEEE Trans. Smart Grid, vol.PP, no.99, pp.1-1

[16] Biegel, B.; Hansen, L.H.; Andersen, P.; Stoustrup, J., "Primary Control by ON/OFF Demand-Side Devices," Smart Grid, IEEE Transactions on , vol.4, no.4, pp.2061,2071, Dec. 2013

[17] Weckx, S.; D'Hulst, R.; Driesen, J., "Primary and Secondary Frequency Support by a Multi-Agent Demand Control System," Power Systems, IEEE Transactions on , vol.PP, no.99, pp.1,11.

[18] Luciano M., Luca R., Helfried B., Chris C., Andrei Z M., Seppo H. and Carlo T., "ELECTRA IRP approach to voltage and frequency control for future power systems with high DER penetrations", CIRED 2015.

[19] Kok, K., Roossien, B., MacDougall, P., van Pruissen, O., Venekamp, G., Kamphuis, R., Laarakkers, J. and Warmer, C., "Dynamic pricing by scalable energy management systems - Field experiences and simulation results using PowerMatcher", Power and Energy Society General Meeting, 2012 IEEE, vol., no., pp.1,8, 22-26 July 2012.

[20] R. Ian and T. Murray, "Domestic electricity demand model". [Online] Available: https://dspace.lboro.ac.uk/2134/5786.

[21] Roscoe, AJ, Mackay, A, Burt, GM \& McDonald, JR 2010, 'Architecture of a network-in-the-Loop environment for characterizing $\mathrm{AC}$ power system behavior' IEEE Transactions on Industrial Electronics, vol 57, no. 4 , pp. $1245-1253$. 